Institut der Universität Bonn um Prüfung einer Milchprobe bat: der Fıemdling wurde in ungnädiger Form abgewiesen. Grieß dachte nicht an die technische Ausnützung seiner Azofarben, und als er endlich begriffen hatte, wieviel Geld andere damit verdienten, und er ein Patent anmeldete, waren es lauter färberisch wertlose Kombinationen. v. Baeyer war der chemischen Industrie abgeneigt, trotzdem er sie so stark beeinflußt hat. Das große Gegenbeispiel allerdings ist Liebig, dessen praktischer Sinn aus seinen, „Chemischen Briefen“ hervorleuchtet. Indessen war ihm die Technik nicht Selbstzweck, und er äußerte zu einem seiner Schüler: „Ich habe mich nie mit Geldverdienen befaßt, das Geld ist mir nur stets nachgelaufen."

Derartige Züge aus dem Wirken unserer Meister zeigen eine Haupteigenschaft des deutschen Charakters: die aus dem Humanismus geborene Hinneigung zur Wissenschaft in ihrer reinsten und idealsten Form. Dieser Idealismus hat Schätze des Geistes geschaffen, die in den Händen der Techniker zu wirklichem Golde wurden. Deshalb stieß Fischer, als er seine Zeitschrift begründete, auf das Vorurteil, daß die theoretische Chemie das Primäre und einzig Vornehme sei, und die angewandte Chemie darum auch literarisch erst in zweiter Linie zu stehen habe. Fischer hat darunter gelitten. Die Vorrede seines Handbuches der chemischen Technologie (1900) ist voll Bitterkeit.

Das war die Stellung der angewandten Chemie in der alten Zeit. Daß eine neue sich vorbereitete, ergibt sich schon aus den Arbeiten Emil Fischers, denn der Erfinder des Veronals, Sajodins und Elarsons war stets bestrebt, einem Teil seiner Forschungen eine praktische Richtung zu geben. Und vollends ist ein Umschwung durch die Kriegsjahre gekommen. Wir empfinden heute, daß die weltabgewandte Arbeit der großen Chemiker des vorigen Jahrhunderts nur auf dem Boden eines politisch aufsteigenden Landes in die deutsche Volkswirtschaft einmünden konnte. Hätten damals so wie heute unsere Feinde gesiegt, so wären wir trotz genialer wissenschaftlicher Theorien wohl kaum zur industriellen Höhe gelangt. Für die NurTheoretiker heißt es darum heutzutage, sofern sie am Aufbau Deutschlands mitarbeiten wollen: Adora quod cremasti! Zwar beruht das technische Gelingen auf der Erkennung der Naturkräfte, und dieses fließt aus rein wissenschaftlicher, von keinem Endzweck eingeengter Forschung; darum wird letztere stets der tiefste und ergiebigste Quell alles Erfolges sein. Aber damit ist nicht gesagt, daß die Wissenschaftler nicht wenigstens im Unterbewußtsein an praktische Ziele zu denken brauchen. Sie müssen es in heutiger Zeit, weil in Deutschland aus volkswirtschaftlicher Ursache die angewandte Chemie eine Bedeutung erlangt hat wie nie zuvor, was auch in der Grundung zahlreicher Forschungsinstitute zum Studium von Kohle, Metallen, Textilstoffen und anderer wichtiger Dinge zum Ausdruck kommt.

Aus dieser Erwägung ergibt sich die Aufgabe der Zeitschrift für angewandte Chemie: sie soll die Befruchtung der deutschen Volkswirtschaft durch chemische Arbeit fördern und beschleunigen, indem sie alles Wesentliche aus Theorie und Praxis zum geistigen Allgemeingut macht. Nach diesem Zweck gliedern sich unsere Veröffentlichungen:

Die Originalbeiträge bringen in Ergänzung der bekannten rein wissenschaftlichen Zeitschriften Experimentaluntersuchungen technischen Inhalts. Das geschieht zwạ schon durch eine Reihe guter Fachblätter aus verschiedenen Zweigen der angewandten Chemie. Aber diese allein genügen nicht. Sie werden meistens nur von den Spezialisten des betreffenden Sonderfaches gelesen, die dadurch in die Gefahr einseitiger Orientierung geraten, und die Mehrzahl der übrigen Chemiker erfährt von dem Inhalt nicht mehr, als die referierende Literatur bietet. Aus diesen Gründen bedarf es des Gegengewichtes eines Sammelorganes, welches aus allen Zweigen der angewandten Chemie das Wichtige und Ausgereifte bringt und es dadurch zu einer Quelle der Anregung für die Gesamtheit der Fachgenossen macht.

Die Fortschrittsberichte gelten der Technik sowohl wie der reinen Wissenschaft. Die Schriftleitung wird bemüht sein, Áutoren zu gewinnen, welche kritisch referieren und das Unwesentliche auszuschalten wissen. Somit soll dem Leser Gelegenheit geboten werden, sich rasch und mühelos über die Weiterentwicklung von Gebieten zu unterrichten, zu deren eingehendem Studium ihm die Zeit fehlt. Darüber hinaus sind namentlich die technischen Berichte dazu bestimmt, vor aller Augen die Probleme zu entrollen, an deren Lösung Interesse besteht; und wenn auch jeder für sich tätig ist, so strömt doch aus dem gemeinsamen Wissen und Erkennen eine Gemeinschaft des Schaffens, welche von jeher der Stolz und die Stärke deutscher Chemiker war.

Die Berichte über die Zusammenkünfte chemischer Gesellschaften', insbesondere innerhalb unseres Vereins, werden wie bisher Rhythmus und Pulsschlag des chemischen Lebens erkennen lassen. Die alten Mitglieder des Vereins deutscher Chemiker mögen danach trachten, neue zu werben, und zwar in dem Bewußtsein, daß es sich nicht nur um eine rein fachliche Sonderbestrebung handelt, sondern um einen Zusammenschluß zur Arbeit an der Wiederaufrichtung Deutschlands.

Dr. Arthur Binz, o. Professor der Chemie an der Landwirtschaftlichen Hochschule Berlin.

\section{Über einige neue Arbeitsmethoden im Gebiete der organischen Chemie.}

Von J. v. Braun.

(Vortrag. gehalten am 7. 10,21 in Dusseldorf bei den Veranstaltungen der Stautlichen Hauptstelle fitr den naturwissenschaflichen Unterricht.)

(Eingeg. 14./12. 1921.)

Das letzte Dezennium hat uns einen derartigen Aufschwung der allgemeinen Chemie beschert, daß daneben die Fortschritte auf dem speziellen Gebiet der Chemie des Kohlenstoffs, die den vorhergehenden Jahrzehnten im wesentlichen ihren Stempel aufgedrückt hatten, ein wenig in den Schatten treten. Eroberungen von solcher Tragweite, wie die Neugestaltung des periodischen Systems nach den Ordnungszahlen der Elemente, die Auffindung und fast restlose genetische Verknüpfung der radioaktiven Elemente, endlich, als letzter Triumph, das Auffinden der Isotopen einer Reihe von Elementen, d. h. die Erschütterung der Lehre von der Konstanz des Atomgewichts eines bestimmten, chemisch und physikalisch wohldefinierten Elements, solche Eroberungen haben wir im Gebiete des Kohlenstoffs nicht zu verzeichnen. Es scheint auch, daß eine so glanzvolle Periode, wie die an die Aufstellung der Kekuleschen Theorie der aromatischen Verbindungen sich anschließenden Jahre, wo Schlag auf Schlag neue Beobachtungen von wissenschaftlich und technisch größter Tragweite gemacht wurden, nicht so bald wiederkommen wird. Immerhin, die 
Summe der neuen Einzelentdeckungen ist eine gewaltige, und vieles ist darunter, was die Keime einer wichtigen weiteren Entwicklung in sich trägt.

Wie in fast allen $/$ weigen der experimentellen Naturwissenscbaften kann man in der modernen Chemie des Kohlenstoffs rech scharf zwei herrschende Arbeitsrichtungen herausschälen: entweder man geht von einer vorgefaßiten theoretischen Vorstellung aus und sucht sie experimentell zu stutzen; dabei kommt man oft in die Lage neuartige Stoffe darzustellen, vorhandene Arbeitsmethoden abzuändern, neue Arbeitsmethoden zu ersinnen. Oder man findet, dank einer scharfen Beobachtungsgabe - in Laienkreisen spricht man dann manchmal in nicht recht zutreffender Weise von einem Zufall - experimentell etwas Neues, verfolgt dann den Gegenstand, vertieft die Beobachtung und wird so in den Stand gesetzt, mit der neuen Waffe einem theoretischen Problem zu Leibe zu rlucken, das mit den alten Waffen nicht anzupacken war. Auf der einen Seite also Vervolikommnung der Arbeitsweise durch einen theoretischen Impuls, auf der andern Vertiefung und Weiterentwicklung der Theorie dank einer neuen Arbeitsmethodilk. So fuhrte im Jahre 1865 Baeyer die wichtige Methode der Zinkstaubdestillation in der Chemie ein, als er bestrebt war, eine Substanz $\mathrm{C}_{5} \mathrm{H}_{7} \mathrm{~N}$, die er, von theoretischen Vorstellungen ausgehend, fur die Muttersubstanz des Indigos hielt und fúr die er im voraus den Namen Indol geprägt hatte, zu fassen, so diente auf der anderen Seite das Phenylhydrazin, das die Meisterhand E. Fischer's entdeckte, dank seiner besondern Reaktionsfähigkeit gegenüber bestimmten Stoffen dazu, um das wichtige Kapitel der Zucker und Hand in Hand damit die Theorie der stereochemischen Verhältnisse ausbauen zu helfen.

Ohne theoretische Vorstellungen kann ein Zweig der Naturwissenschaft von so reichem Inhalt, wie es die organische Chemie ist, nicht auskommen; wir brauchen sie, um uns im Gewirr der Tatsachen zurechtzufinden, und sie verhelfen uns, im Sinne der einen oben angedenteten Arbeitsrichtung zu neuen Fortschritten. Aber sie sind nicht das bleibende: sie führen oft nur ein kurzes Dasein sterben ganz $a b$, oder zum mindesten verändern sich weitgehend. Was aber bleibt und nie seinen Wert verliert, das ist eine richtige Beobachtung. Ind so habe ich vorgezogen, Ihnen heute aus mejnem speziellen Arbeitsgebiet nicht über eine Reihe neuerer theoretischer Vorstellungen einiges vorzutragen, sondern Sie mehr mit experimentellen Errungenschaften bekannt zu machen, die die organische Chemie der letzten Jahre aufzuweisen hat. Ich kann dabei natürlich lange nicht alles berücksichtigen und nuß eine gewisse Auswahl treffen.

Die vollständige Untersuchung eines organischen Stoffes umfaßt drei Teile: 1. durch die Analyse bestimmen wir seine Zusammensetzung; 2. durch die Molekulargewichtsbestimmung seine Teilchengröße; 3. durch Umwandlungen verschiedenster Art - es können Reaktionen des Abbaues oder Aufbaues und sonstige Untersuchungen seines Verhaltens sein - stellen wir fest. wie der Stoff aus den Elementaratomen zusammengefügt ist. Die vielseiligsten Fortschritte hat die organische Chemie in bezug auf den letzten Punkt in neuerer Zeit gemacht, aber auch der erste und zweite Punkt weisen eine sehr wesentliche Weiterentwicklung auf: ich will sie als Mikronisierung der Operationen bezeichnen.

Die organische Mikroanalyse, deren Ausarbeitung wir hauptsächlich dem österreichischen Chemiker Pregl verdanken, ist prinzipiell von der gewöhnlichen, seit vielen Jahrzehnten gebräuchlichen organischen Analyse nicht verschieden, nur arbeitet sie im kleinsten AusmaB: Kohlenstoff und Wasserstoff werden im Sauerstoffstrom mit Hilfe von Kupfer oxyd und Bleichromat verbrannt und die Verbrennungsprodukte wie nach Liebig aufgefangen, der Stickstoff wird nach dem Verfahren von Dum as volumetrisch bestimmt; wảhrend aber bisher Dezigramme Substanz genommen wurden, wird bei der Mikroanalyse die Menge in Milligrammen ausgedrückt. Dementsprechend sind alle apparativen Einrichtungen weitgehend verkleinert, und die Wage, die man benutat - sie wird heute $z$. B. von Sartorius in Göttingen, dann von Ku hl ma $n \mathbf{n}$ in Hamburg geliefert und heißt allgemein Mikrowage -, erlaubt ohne besondere Schwierigkeit, mit der Genauigkeit bis auf herunterzugehen. Die Vorteile der organischen Mikroanalyse, die sich in wenigen Jahren die Welt erobert hat und kaum in einem der größeren rbemischen Institute noch fehlt, bestehen zum Teil in Gasund Zeitersparnis, vor allem aber in einer gewaltigen Materialersparnis Und das ist nun wiederum deshalb sehr wichtig, weil sich in neuerer Zeit die organische Chemie immer mehr der Bearbeitung biochemischer Vorgänge zuwendet und der Experimentator sehr hăufig nur minimale Mengen wertvoller, aus dem Pflanzen- und Tierleib isolierter Substanzen zu fassen bekommt. Eine Reihe wichtiger Untersuchungen der neuesten Zeit hätten ohne das Hilfsmittel der Mikroanalyse (ich möchte als Beispiele an die Untersuchung des Krötengifts von Wieland und der Nukleinsäuren von Levene erinnern) vielleicht kaum ausgeführt werden können.

Etwas weniger als die Mikroanalyse hat sich bis jetzt noch die Mikronisierung der Molekulargewichtsbestimmung eingebürgert. Die gewöhnlichen ebullioskopischen und kryoskopischen Methoden kann man zwar auch unter Beachtung gewisser Kautelen kleinen Mengen anpassen, wichtiger und aussichtsvoller scheint mir aber eine in der Ausführung verblüffend einfache Methode zu sein, die wir Barger (L,ondon) verdanken: sie beruht auf dem einfachen Prinzip, daß aus einer verdünnten Lösung das Lősungsmittel bestrebt ist, zu einer konzentrierteren überzudestillieren, um die Konzentrationen auszugleichen.
Stellen wir uns vor, wir hätten in eine Kapillare zwei Tropfen A und $B$ hineingebracht und unter dem Mikroskop unmittelbar hinterher die Durchmesser mit Hilfe einer eingeritzten skala festgestellt: sind die I.obsungen A und B äquimolar, so wird sich nichts ändern, wenn nicht, so wird $A$ oder $B$ in der Taille ditnner werden. Wir brauchen also, um die molare Konzentration einer unbekannten Losung $\mathrm{B}$ zu finden - und daraus in bekannter Weise das Molekulargewicht abzuleiten -, nichts weiter, als in mebreren Kapillaren Tröpfchen der Lossung $B$ mit Tröpfchen von Standardlosungen

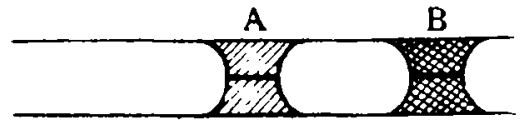

A von wechselnder, passend abgestufter molarer Konzentration zusammenzubringen, bis diejenige Kombination gefunden ist, bei der die 'Tröpfchen unverändert bleiben. Kleine Verbesserungen dieser sehr schonen und einfachen Methode, die vor wenigen Tagen aus dem Wirzburger Laboratorium mitgeteilt worden sind ${ }^{1}$ ), berechtigen $z u$ der Erwartung, daß sie bald der Mikroanalyse als ebenbiurtig zur Seite treten wird.

Gewissermaßen als Mikronisierung konnen wir weiterhin eine Reihe von Errungenschaften bezeichnen, die wir auf dem Gebiet der Anwendung physikalischer Faktoren zu chemischen Zwecken antreffen.

Bei der Untersuchung der organischen Stoffe haben seit langem schon optische Verhältnisse eine bedeutsame Rolle gespielt: aus der Fähigkeit, Licht einer bestimmten Nuance zu absorbieren, oder einen Lichtstrahl in bestimmter Weise zu brechen, aus der Fähigkeit, polarisiertes licht abzulenken, lernte man Schlüsse auf den inneren Bau des organischen Moleküls zu ziehon. Was aber dabei als "I Iicht" benutzt wurde, das war immer nur der kleine Teil von Wellen $[0,4$ bis 0,8 " $]$, fur die zufällig unser Auge empfindlich ist.

Ganz allmählich lat man sich nun von diesen Schranken freigemacht und hat sie nach unten ubberschritten; erst waren es die ultravioletten Strahlen, dann die allerkürzesten, die Röntgenstrahlen, die in den Dienst der organischen Forschung gestellt wurden.

DaB ultraviolette Strablen genau so wie sichtbare in selektiver Weise absorbiert werden konnen, sagte schon 1862 Miller. Etwas später (1879) setzte diese Untersuchungen Hartley fort, der auch die Arbeitsmethode entwickelt hat. Sie besteht darin, daß eine an ultravioletten Strablen reiche Lichtquelle $z$. B. ein Fisenlichtbogen oder eine Quecksilberdampflampe vor dem Spalt eines Spektralapparates in Gang gesetzt wird. Das Strablenblundel wird durch ein Prisma zerlegt, passiert dann die Substanz, die auf ihr Absorptionsvermögen geprüt werden soll, und fällt auf eine photographische Platte, die nach der Entwicklung sehr genau die stattgefundene Absorption abzulesen gestattet. Trotzdem num diese Arbeitsmethode, an der einige sehr wesentliche Verbesserungen in letzter Zeit von V. Henri angebracht worden sind, seit einer Reihe von Jahren gegeben war, hat man verhältnismäßig weuig mit ihr angefangen. Erst in neuerer Zeit wandte man sich ihr wieder zu. Unfangreiche Untersuchungen, die zum Teil in Hartleys Heimat (England), vor allem aber in Leipzig vón $\mathrm{H}$ antzs $\mathrm{ch}$ und seiner Schule ausgeführt worden sind, haben gezeigt. eine wie außerordentlich wertvolle Ergănzung dessen, was man im sichtbaren licht beobachtet, gewonnen wird, wenn man die Beobachtungen auf die kurzwelligeren ultravioletten Strahlen ausdehnt.

Insbesondere gilt das natürlich für Substanzen, die für unser Auge farblos oder ganz schwach farbig erscheinen, und das sind die verhảltnismảßig einfach gebauten organischen: wird das Molekül großßer, komplexer, so verschiebt sich die Absorption in der Richtung der langen Wellen, es tritt demnach eine rote, gelbe, grüne, blaue, schließlich violette Farbe auf, die bei einfach gebauten organischen Stoffen meist fehlt. Zu solchen farblosen Substanzen, die aber im Ultraviolett recht kräftig selektiv absorbieren, gehort unter anderm das Benzol, die Grundsubstanz unserer Teerfarbstoffe. Ohne hier auf die Unmenge von Einzelergebnissen, die auf diesem Gebiet bisher schon gewonnen wurden, einzugehen, möchte ich nur darauf hinweisen, daß die Untersuchung der ultravioletten Absorption namentlich im Gebiete tautomerer, sich sehr leicht isomerisierender Substanzen wertvolle Dienste Jeistet, und will an einem ganz einfachen Beispiel zeigen, zu wie überraschenden Ergebnissen man bei Anwendung dieser Methode in scheinbar völlig klaren Fällen kommt.

Wenn wir eine beliebige organische Carbonsäure $\mathrm{R} \cdot \mathrm{CO}_{2} \mathrm{H}$ ins Auge fassen, dann pflegen wir ibr dieselbe strukturelle Formel $R \cdot C^{\prime \prime}$ zu erteilen, wie ihren Salzen R.C $C^{\circ} \mathrm{O}$-..Me und ihren Estern $\mathrm{O}^{\mathrm{O}}$

$\mathbf{R} \cdot \mathbf{C}^{1} \cdot \mathbf{O} \cdot \mathbf{R}^{\prime}$. Der einzige seit der Begrïndung und Durchführung der elektrolytischen Dissoziationstheorie uns bekannte Unterschied besteht darin, daß in der Säure und dem Salz die Bindung zwischen $O$ und $H$ oder Me in bestimmten Lösungsmitteln, vor allem im Wasser, gelockert ist, was aber die gesamten Bindungsverhältnisse, insbesondere den Komplex - $-\mathrm{C}^{\mathrm{II}}$. nicht berührt. Da nun für die Absorption der Grad der Dissoziation oder Assoziation von Molekulen ohne EinfluB ist, da
ferner das Wasserstoffatom, ein optisch indifferentes Alkali- oder Erd-

1) Rast, Her. 54, $1979[1921]$ 
alkalimetallatom und endlich ein gesättigter Alkylrest sie kaum beeinflukt, so sollte man meinen, daß3 nur minimale Verschiedenheiten der Absorption bei Säuren, Salzen und Estern auftreten wïrden. Die optische Untersuchung zeigt nun, daB die Absoıptionsverhältaisse im Ultraviolett ganz unerwartete Unterschiede zeigen:

1. Alle Ester absorbieren untereinander gleich ohne Rücksicht auf die Größe von $\mathbf{R}^{\prime}$;

2. Alle Salze (insbesondere Alkali und Erdalkalisalze) absorbieren untereinander gleich, aber verschieden von den Estern und zwar schwächer als diese;

3. Bei Säuren finden wir kleine Schwankungen, allgemein aber liegt ihre Absorption zwischen der von Salzen und Estern.

Bezigglich der Ester steht fest, daß sie die_oben angeführte Formel $\mathrm{R} \cdot \mathrm{C} \searrow_{O R}^{\mathrm{O}}$ haben; wenn die Säuren und Salze versçhieden absorbieren, so muß - das lehren uns alle Erfahrungen der Spektroskopie - in ihrem Molektul eine Verschiedenheit bezüglich der Bindungsverhältnisse vorliegen, und da die Dissoziation allein keine Erklärung abzugeben vermag, bleibt als einzige Erklärungsmöglichkeit die, daß der Wasserstoff und die Metalle nicht wie die Alkylreste an e in em Sauerstoffatom sitzen, sondern sich in der Anziehungssphäre der beiden Sauerstoffatome befinden: $\mathrm{R} \cdot \mathrm{C}<\mathrm{O}$ ) $\mathrm{Me} \mathrm{R} \cdot \mathrm{C}<\mathrm{O}_{\text {o) }}^{\mathrm{O}} \mathrm{H}$, eine Formulierung, die bereits über das hinausgeht, was unsere Valenzformeln auszudrücken vermögen, die aber in den Kreis der theoretischen Vorstellungen, wie sie für das Gebiet der anorganischen Verbindungen von A. Werner entwickelt wurden, gut hineinpaßt. - Bei den Salzen herrscht offenbar der eigenartige Bau des Moleküls restlos vor, die Säuren mit ihrer mittleren Absorption schließen sich teils den Salzen, teils den starrgebauten Estern an $\left[\mathrm{R} \cdot \mathrm{C} / \mathrm{O}^{\mathrm{O}} \mathrm{H}\right.$ und $\left.\mathrm{R} \cdot \mathrm{C} / \mathrm{OH}_{\mathrm{OH}}^{\mathrm{O}}\right]$ : das ist plausibel, denn wenn der Wasserstoff auf der einen Seite als Analogon der Alkalimetalle erscheint, so stellt er auf der anderen Seite das Endglied in

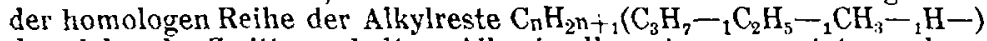
dar; daher das $\mathrm{Zwitterverhalten.} \mathrm{Alles} \mathrm{in} \mathrm{allem} \mathrm{ein} \mathrm{unerwartetes} \mathrm{und} \mathrm{un-}$ gemein interessantes Ergebnis.

Wie die ultravioletten Strahlen, so könnten und mußten selbstverständlich auch die ultraroten Strahlen von größerer Wellenlänge herangezogen werden, um das Bild der Absorption zu vervollständigen, ja die neue Iehre vom Aufbau des chemisehen Atoms aus dem positiv geladenen winzig kleinen Kern und den negativ geladenen Elektronen läßt gerade bei den ultraroten Strahlen ganz besonders interessante und wichtige Resultate erwarten. Leider ist die Arbeitsmethodik auf diesem Gebiet noch sehr wenig entwickelt und was bisher geleistet wurde, ist an Umfang gering; die Weiterentwicklung gerade dieses Kapitels, die natiulich nur von physikalischer Seite erfolgen kann, dürfte aber für die Chemie der Kohlenstoffverbindungen von größter Bedeutung werden.

Stellt nun die Anwendung der ultraroten Strahlen einstweilen noch ein Zukunftsbild dar, so ist es in allerletzter Zeit gelungen, das Endglied der Wellenskala, nämlich die Wellen mit kleinster Länge, die Röntgenstrahlen in den Dienst der organisch-chemischen Forschung zu stellen. Die Sache ist zwar erst im Werden, eroffnet aber so wichtige Ausblicke für später, daß ich mir nicht versagen kann, sie hier zu erörtern.

Wenn man, wie es zuerst vor 9 Jahren v. Laue mit seinen Mitarbeitern Friedrich und Knipping gezeigt hat, Rontgenstrahlen durch eine Kristallplatte hindurchfallen läßt, oder wie bald darauf die Pbysiker Bragg zeigten, von einer Kristaljplatte reflektieren läBt und auf einer photographierten Platte auffängt, so erhält man kleine, in bestimmter Weise angeordnete Flecke, die von Beugungs- und Interferenzerscheinungen herrühren und in engster Beziehung zum Raumgitter stehen, nach welchem der Kristall aufgebaut ist. Ein noch einfacheres Verfahren, welches wir den Physikern Debye und Scherrer verdanken, ermöglicht die Verwendung von Kristallpulver und zwar in außerordentlich kleiner Menge. Es ist ferner gelungen, durch rechnerische Auswertung der photographischen Bilder nicht nur das System, dem der kristallisierte Korper angehört, zu ermitteln, sondern auch die absoluten Abmessungen dieses Elementarkörpers zu bestimmen unter Elementarkörper oder Elementarparallelepiped versteht man den kleinsten Teil eines Kristalles, der bereits alle Symmetrieejgenschaften des Kristalles zeigt; der Gesamtkristall wird also lediglich durch periodische Wiederholung des Elementarkörpers in stets zu sich selbst paralleler Lage nach allen drei Dimensionen des Raumes gebildet.

Mit diesem Hilfsmittel ging man nun in letzter Zeit - und zwar war es Herzog mit seinen Mitarbeitern im Kaiser Wilhelm-Institut für Faserstoffchemie in Berlin-Dahlem - an die Untersurhung einer Reihe ron hochmolekularen organischen Substanzen, hauptsächlich von biologischem Material: Cellulose, Stärke, Glykogen, Seide, Kunstseide, Wolle, menschliches Haar gelangten zur Untersuchung und ergahen sehr bemerkenswerte Resultate: erstens stellte sich heraus, daß während ein Teil dieser hochmolekularen Substanzen in Einklang mit dem, was man bisher annahm, zweifellos amorph ist - das trifft z. B. zu bei Wolle, bei Haaren, bei Glykogen - erweisen sich Seide Cellulose und Stärke überraschenderweise zweifellos als kristallisiert. Man kann aber noch einen Srhritt weiter tiber die Feststellung dieser Tatsache hinausgehen und folgende rein ebemische Tatsache ableiten.
Die Cellulose zerfällt bekanntlich bei der Hydrolyse genau so wie Stärke in Traubenzuckermoleküle, die man daber bisher als die eigentlicben Bausteine der Cellulose auffafte. Wenn man aber bei der Hydrolyse vorsichtig verfährt, so gelingt es zum großen Teil (35-60\%, je nach den Arbeitsbedingungen) je zwei Traubenzuckermoleküle verbunden zu erhalten und eines der vielen bekannten Disaccharide, die sogenannte Cellobiose zu fassen. Aus den Abmessungen des Elementarkörpers des Cellulosemoleküls ergibt sich, daß er aus vier Traubenzuckerresten besteht, und da die Cellulosekristalle der hemimorphen Gruppe des monoklinen Systems angehoren, folgt aus kristallographischen Symmetrjegründen, daß jeder Elementarkörper aus je zwei Doppelzucker - d. h. Cellobioseresten bestehen muB, d. h. die ganze - Cellulose muß aus Cellobiose aufgebaut sein und $z$ war so, daß Komplexe von zwei miteinander verknüpften Cellobioseresten sich $n$-fach im Molekull wiederholen. Die alte schematische Darstellung der Strukturformel der Cellulose (worin $Z$ einen Traubenzuckerrest bedeuten möge)

$(Z-Z-Z-Z-Z \ldots) 4$
wird $z u$ der schon mehr aussagenden Formel

$$
\{[(Z-Z)(Z-Z)][(Z-Z)(Z-Z)][(Z-Z)(Z-Z)] \ldots\}_{n}
$$

aufgelöst und die Röntgenoptik läßt so eine Entscheidung über die Bausteine eines großen Moleküls zu! Nur mit größter Spannung kann man weiteren Untersuchungen mit dieser ganz neuen Arbeitsmethode entgegensehen.

Ich erwähnte vorhin, daß der Übergang von sichtbarem Licht zu ultraviolettem scböne Erfolge im Gebiet der Konstitutionsbestimmung organischer Verbindungen gezeitigt hat. Seit langem weiß man, daß Lichtenergie auch zur Förderung chemischer Umsetzungen dienstbar gemacht werden kann und daß die Wirksamkeit der Strahlen fast ausnahmslos mit abnehmender Wellenlänge zunimmt. Was liegt da näher als photochemische Umwandlungen im organischen Gebiet durch Anwendung von an ultravioletten Strahlen rejchem Licht au förden? Merkwürdigerweise sind Untersuchungen nach dieser Richtung bis jetzt erst spärlich ausgeführt worden; sie haben aber, wo die Methode Anwendung fand, zu interessanten Resultaten geführt und werden zweifellos im Laufe der Zeit eine große Bedeutung im Gebiete der Chemie des Kohlenstoffs erlangen. Aus dem bisher vorliegenden Material möchte ich zwei Tatsachen hervorbeben, die wir beide dem Rostocker Chemiker Störmer und seinen Schülern verdanken.

Erstens: Bekanntlich können Verbindungen mit einer Doppelbindung in geometrisch isomeren Modifikationen existieren, von denen eine einem stabileren, die andere einem labileren molekularen Aufbau entspricht. Wohl am bekanntesten unter solchen Isomerenpaaren sind die zwei Zimtsäuren

$$
\begin{array}{cc}
\mathrm{C}_{6} \mathrm{H}_{5}-\mathrm{C}-\mathrm{H} & \mathrm{C}_{6} \mathrm{H}_{5} \cdot \mathrm{C}-\mathrm{H} \\
\mathrm{CO}_{2} \mathrm{H}-\mathrm{C}-\mathrm{H} & \mathrm{H}-\mathrm{C} \cdot \mathrm{CO}_{2} \mathrm{H} \\
\text { Sup. } 42^{\circ} & \text { Sup. } 133^{\circ}
\end{array}
$$

von denen die höher schmelzende stabile sehr leicht $\mathrm{zu}$ fassen ist, während das Gegenteil für die labile galt, deren Isolierung und Untersuchung jahrelang den Gegenstind schwieriger, scharfsinniger und sorgfältiger Untersuchungen gebildet hatte. Daß durch Licht ein labiles geometrisches Isomeres sich in das stabile umlagern kann, war bekannt. Störmer zeigte nun, daß bei genügender Energiezufuhr durch ultraviolettes Licht auch der umgekehrie Vorgang realisiert werden kann und so war mit einem Scblag ein Weg gewiesen, um mit leeichtigkeit in den Besitz dieser recht interessanten labilen Stoffe zu gelangen. Es ist kaum daran zu zweifeln, daß die Metbode auch im Gebiete anderer Stoflklassen sich wird anwenden lassen und wer weiß, ob sie nicht auch im Gebiete physiologisch wirksamer Substanzen zu Frfolgen fahren wird. Andeutungen dafur scheinen, wie mir von befreundeter medizinischer Seite mitgeteilt wurde, vorhanden zu sein. Ein zweites, praktisch auch wichtiges, vielleicht bei weiterer Verfolgung des Gegenstandes noch wichtigeres Resultat ist gleichfalls von Störmer mit Hilfe der kurzwelligen ultravioletten Strahlen erzielt worden: es lassen sich damit organische Säuren mit Alkoholen verestern. Der Gedanke liegt nahe, daß die so zahlreich in der Pflanzenwelt vorkommenden Ester diesem Faktor - Sonnenlicht enthält ja auch ultraviolette Strahlen - ibre Entstehung ver danken, und nicht von der Hand za weisen ist die Annahme, dab diese und andere noch sicher aufzufindende Aufbaureaktionen vielleicht zu technisch wichtigen Darstellungsverfahren sich werden ausbauen lassen.

Wie bei der strahlenden Energie die Fortschritte der letzten Zeit charakterisiert sind durch Mitherürksichtigung von Wellen kleinerer Grobe, so finden wir diese "Mikronisierung" - um den einmal gebrauchten Ausdruck hier wieder zu benutzen - auch bei einem weiteren physikalischen, für das praktische Arbeiten ungemein wichtigen Faktor, nämlich dem Druck. Die Vorteile der Verwendung niedrigen Druckes - namentlich zur Reinigung der Substanzen durch Trocknen, Überdestillieren, Sublimieren - kennt man schon lange. Die einfache von Bunsen ersonnene apparative Vorrichtung, die Bunsensche Wasserstrahlpumpe, die $10-15 \mathrm{~mm}$ Quecksilber liefert, war lange Zeit die einzige Vorrichtung die im Durchschnittslaboratorium zur Verfügung stand. ÖI- oder Quecksilberpumpen mit einem Wirkungsgrad bis zu Bruchteilen eines Millimeters Quecksilber waren unverhältnismäßig teuer und daher nur spärlich vertreten. Seit einigen 
Jahren hat sich das Bild vollig verändert: die sinnreichen kleinen Apparate, z. B. die Pumpen von Vollmer und Friedrich, die bandlich und billig sind und selbst bei den heutigen Etatsverhältnissen ihre Anschaffung einem Durchschnittsinstitut gestatten, sind im allgemeinen Gebrauch, und ihre Verwendung ermöglicht die Reindarstellung einer ganzen Reihe von Stoffen in schneller und einfacher Weise. Viele der in letzter Zeit isolierten Verbindungen (z. B. das Phytol, einer der Bestandteile des Chlorophylls, die höhermolekularen Bausteine des Eiweißmoleküls) wären ohne Anwendung stark erniedrigten Druckes kaum zu fassen gewesen. Direkte chemische Umsetzungen bei vermindertem Druck sind noch spärlich, zeigen aber schon in einer Ricbtung einen theoretisch wohl vorauszusehenden Erfolg: die Depolymerisation von gasförmigen Molekülen, die durch kurze Berührung mit einer Wärmequelle (z. B. einem glühenden Metalldraht) erreicht werden kann, wird durch Minderdruck befördert: das zeigen $z$. B. die Versuche von Harries und Sta u dinger über die Spaltung von Terpenkohlenwasserstoffen $\mathrm{C}_{10} \mathrm{H}_{16}$ in Molektile des Isoprens $\mathrm{C}_{5} \mathrm{H}_{5}$, das auch aus Kautschuk durch thermischen Zerfall hervorgehen kann und umgekebrt wieder zu Kautschuk sich polymerisieren läßt. Einfache Gesetze für diesen und ähnliche Vorgänge der technisch so wichtigen and daher mannigfach studierten Polymerisation haben sich bis jetzt tbrigens noch nicht herausschälen lassen.

Wie in der anorganischen Chemie, so spielen auch in der organischen Umsetzungen, die durch Gegenwart kleiner Mengen von Fremakörpern weitgehend beschleunigt werden, seit jeher eine besonders wichtige Rolle: beruht doch bekanntlich der stoffliche Umsatz im Tier- und Pflanzenreich zum weitaus größten Teil auf der Mitwirkung solcher Katalysatoren, die man hier als Fermente oder Enzyme bezeichnet, und man kennt auch schon längst künstliche stoffliche Umsetzungen, die - wie die bei Gegen wart von Aluminiumchlorid sich abspielende Friedel-Crafts sche Reaktion - einer katalytischen Forderung bedürfen. Die auf diesem Gehiete in neuester Zeit geleistete Arbeit ist von grobter Wichtigkeit Teils hat sie bereits neue gut ganghare Wege des Arbeitens geschaffen, teils solche in sichtbare Nähe gerückt. An die Spitze gestellt zu werden mit Rucksicht auf den Grad des schon Erreichten verdient zweifelsohne die katalytische Hydrierung. Der erste Impuls dazu stammt, wie wir neidlos anerkennen wollen, von dem französischen Chemiker Sabatier, welcher zeigen konnte, daß kleine Mengen fein verteilten Nickels Wasserstoff, wenn er mit Dämpfen organischer für Wasserstoff aufnahmefähiger Substanzen in Berübrung kommt, seine Anlagerung an die organische Substanz veranlassen. Die der Sabatierschen Methode zugrunde liegende Idee ist dann vielfach variiert worden. Die deutschen Forscher Paal, Skita, Willstätter erkannten, daß das Nickel auch durch andere Metalle (Pt, Pd) ersetzt werden kann, man fand - auch diese Erweiterung geschah bei uns - , daß Flüssigkeiten ebensogut der Hydrierung zugänglich sind, man stellte fest, daß - wie vorauszusehen war - sehr erhöhter Wasserstoffdruck die Reaktion bedeutend fördert (I patiew). Ungezählte Verbindungen sind in den letzten Jahren nach diesem neuen Verfahren mit Wasserstoff beladen worden: Stoffe mit doppelter und dreifacher Kohlenstoffbindung $\mathrm{C}=\mathrm{C}, \mathrm{C}=\mathrm{C}$ (die sogenannten ungesättigten Verbindungen), mit doppelter $\mathrm{C}=\mathrm{O}$-Bindung (Aldehyde und Ketone), mit $\mathrm{C} \cong \mathrm{N}$-Bindung (Nitrile) und endlich auch aromatiscbe Verbindungen, bei denen ja die Frage, ob sie wirklich drei Doppelbindungen im Kohlenstoffkern enthalten, noch immer nicht einwandfrei beantwortet ist, haben sich als geeignetes Versuchsmaterial erwiesen. In Einzelfällen hat diese Arbeitsmethode sogar schon eine weit uber das wissenschaftliche Interesse herausgehende Bedeutung erlangt. Das gilt insbesondere für die Hydrierung der Fette und die Hydrierung des Naphthalins. Ungesättigte flüssige Fette werden durch Wasserstoffaufladung in wertvollere feste Fette verwandelt, umgekehrt geht festes Naphthalin $\mathrm{C}_{10} \mathrm{H}_{8}$ durch Aufnahme von 4 Atomen Wasserstoff, in flüssiges Tetrahydronaphthalin (Tetralin) $\mathrm{C}_{10} \mathrm{H}_{12}$, von 10 Atomen Wasserstoff in gleichfalls flüssiges Dekahydronaphthalin (Dekalin) $\mathrm{C}_{10} \mathrm{H}_{18}$ über (Schroeter); beide haben gerade wegen ihrer flüssigen Beschaffenheit $z$. B. als Benzinersatz für Motoren, ferner als Terpentinölersatz in der Lackindustrie und für verwandte $Z$ wecke schnell eine Bedeutung erlangt (v. G w in ner, Schrauth), bei beiden, insbesondere beim Tetralin gelingt es durch chemische Umformungen zu großen Scharen von Abkömmlingen zu gelangen, die nach verschiedenen Richtungen, z. B. meinen eigenen noch unveröffentlichten Versuchen zufolge, insbesondere nach pharmakologischer Richtung von Interesse sind. Wie das Nickel imstande ist, unter bestimmten im Einzelfall zu ermittelnden Bedingungen den Wasserstoff zu fixieren, so kann es andererseits auch, und dasselbe tun noch andere Metalle, wie Kupfer, unter etwas abgeänderten Bedingungen, insbesondere bei etwas mehr erhobter Temperatur eine Wasserstoffabspaltung, also eine Dehydrierung bewirken. Im Endeffekt kommt eine Dehydrierung auf eine Oxydation hinaus, und unwillkürlich drängt sich in diesem Zusammenhang die Frage auf, ob es wohl möglich sei, auch den molekularen Sauerstoff durch katalytische Beeinflussung zur Reaktion mit organischen Stoffen zu bringen. Die Frage ist mehrfach in Angriff genommen worden: Phosphorverbindungen, Vanadinverbindungen, Osmiumverbindungen wurden auf ihre Wirkung untersucht, einstweilen aber hat sich eine glatte Lobsung noch nicht finden lassen. Sie erscheint auch, vom praktischen Gesichtspunkte aus vielleicht nicht ganz so wichtig wie die der katalytischen Hydrierung, weil ja der Sauerstoff leicht in seine aktivere Modifikation, das Ozon, verwandelt werden kann und in dieser Form nach den vokannten zu Beginn dieses Jahrhunderts ausgeftibrten Untersuchungen können, mit großßter Leichtigkeit angreift.

Etwas besser durchgearbeitet als die katalytische Oxydation sind noch einige andere Umsetzungen, die aber nicht die Bedeutung besitzen wie ale Hydrierung: so die Abspaltung von Wasser, die aus einem Gemisch von Alkohol und Ammoniak bei Gegenwart von Stoffen wie Thordioxyd erfolgt und die Bildung von Aminen zur Folge hat, oder die Zersetzung urganischer Säuren, die teils in einem Austritt vón Kohlendioxyd und Bildung des Kohlenwasserstoffs oder einem Austritt von Kohlendioxyd und Wasser und Bildung eines Ketons besteht.

Von Interesse sind diese letztgenannten Umformungen deshalb, weil sie möglicherweise - neben vielen anderen Umformungen - auch in der organisierten Welt unter dem spezifischen Einfluß von Beschleunigern sich abspielen und uns so zum Kapitel der Katalyse bei biologischen Prozessen herüberleiten. Die Erscheinungen di es e r Katalyse sind ungemein zablreich und ihre Aufklärung besitzt daher die allergrößte Bedeutung. Wenn der Pflanzen- und der Tierleib imstande ist eine Unzahl von Verbindungen auf dem Wege des Aufund Abbaues darzustellen, ohne extreme Bedingungen, wie wir sie im Reagensglas und Autoklaven realisieren, zu Hilfe zu nehmen, so geschieht das eben deshalb, weil ihnen zahllose Fermente zur Verfugung stehen; und es ist kein Zweifel, daß wir an dem Tage unsere komplizierten chemischen Arbeitsmethoden werden abbauen und durch einfachere ersetzen können, wo es uns gelingen wird, hinter das Geheimnis der natïrlichen Fermente zu kommen, sie erstens rein $z u$ fassen, sie zweitens ihrer Natur nach zu diagnostizieren und sie drittens in ihrer Wirkung genau zu erforschen. Was bisher nach dieser Richtung vorliegt, sind trotz massenhafter Arbeit die allerersten Anfänge: wohl war es moglich, aus Organen aktive Stoffe zu isolieren, die, um nur wenige Beispiele herauszugreifen, wie die Lipase Fette in Glycerin und Säuren spalten, wie die Zymase Zucker zur weingeistigen Gärung veranlassen, wie die neuerdings năher untersuchte Tannase, Gerbstoffe in Gallussäure und Zucker trennen, und wie die auch neuerdings studierte Carboxylase aus Sänren Kohlendioxyd entwickeln, wohl sind wir in vielen wichtigen Fällen, so bei der alkoholischen Gärung ( $\mathrm{Neuberg}$ ) imstande, uns ein zutreffendes Bild des chemischen Vorganges zu machen, minimal sind aber erst die Fortschritte bezüglich der Reindarstellung der Fermente und gleich Null bezdiglich ihrer chemischen Charakterisierung. Die Bewältigung dieser Aufgabe bietet unendliche Schwierigkeiten in Anbetracht der Leichtveränderlichkeit der fraglichen Stoffe, und sie muß unter subtilen Bedingungen und mit Vorsichtsmaßregeln in Angriff genommen werden, die uber das bisher in der organischen Chemie gewohnte Maß weit hinausgehen. Sorgfältigste Abstufung der sauren oder alkalischen Reaktion der Reagentien, Verwendung von spezifischen Adsorptions- und Dialysiermitteln, in jeder Hinsicht behutsamstes Arbeiten - um nur auf einige experimentelle Momente hinzuweisen - sind die Punkte, auf die man zu achten hat, wie dies der Hauptförderer dieser Arbeitsmethodik, R. Willstätter in München, in allerletzten Jahren gezeigt hat. Unendlich groß sind die Schwierigkeiten, die sich hier bieten, aber unendlich groß der Preis, der die Mühe lohnen wird. Denn das Gelingen des Werkes muß der organischen Chemie ganz neue Bahnen weisen. Die, die sie bis -vor kurzem gegangen ist, sind reichlich ausgetreten. Die Ab- und Aufbaureaktionen, die Erkennungsmethoden organischer Stoffe, wie sie die Meister der organischen Chemie - v. Baeyer, Wallach, Fischer, Willstäter u. a. - in den heute als klassisch geltenden Arbeiten über Zucker, Eiweiß, Harnsäure, Pflanzenfarbstoffe, Terpene, Gerbstoffe benutzten, führen über eine gewisse Grenze nicht hinaus. Und daher sehen wir, wie man bestrebt ist, das Handwerkzeug zu vermehren und $z u$ verfeinern und wie man bei diesen Bemutungen - das habe ich im vorhergehenden zu betonen versucht - Anlehnung an verwandte naturwissenschaftliche Gebiete, das der Physik und Kristallographie auf der einen und das der biologischen Wissenschaften auf der anderen Seite, sucht.

\section{Chemische Technologie als Examensfach? \\ Von A. Binz \\ (Eingeg. 30.|11. 1921.)}

Vor einiger Zeit wurde an maßgebender Stelle eine Diskussion der Frage angeregt, ob chemische Technologie als Examensfach zugelassen werden soll. Die Antwort lautete verneinend, da in den Vorlesungen über anorganische und organische Chemie hinreichend Gelegenheit sei, auch das wichtigste aus der Technologie zu bringen, und ferner, weil die Studierenden Technologie doch nur wăhlen würden, um der Prüfung in einem anderen schwierigeren Fache zu entgehen.

Da mein jetziges Lehramt die reine Chemie betrifft, so gerate ich wohl nicht in den Verdacht, pro domo zu reden, wenn ich mich zu dem Gegenstand äußere, der mir andererseits aus fruherer. akademischer Tätigkeit vertraut ist.

Nimmt man das klassische zweibändige Lehrbuch der chemischen Technologie von F. Knapp (1847) zur Hand, daß im ganzen 1550 Seiten umfaßt, und in dem man trotz dieser Fulle kein Wort missen möchte, so erkennt man, daß es ein Irrtum ist zu glauben, man könne chemische Technologie nebenbei in der Vorlesung uber reine Chemie behandeln. Dafür ist der Stoff auch bei Beschränkung auf das Wesentliche zu 